\title{
Detection of glioblastoma in biofluids
}

\author{
Javier M. Figueroa, MD, PhD, and Bob S. Carter, MD, PhD \\ Department of Neurosurgery, University of California, San Diego, La Jolla, California
}

The detection of glioblastoma (GBM) in biofluids offers potential advantages over existing paradigms for the diagnosis and therapeutic monitoring of glial tumors. Biofluid-based detection of GBM focuses on detecting tumor-specific biomarkers in the blood and CSF. Current clinical research concentrates on studying 3 distinct tumor-related elements: extracellular macromolecules, extracellular vesicles, and circulating tumor cells. Investigations into these 3 biological classifications span the range of locales for tumor-specific biomarker discovery, and combined, have the potential to significantly impact GBM diagnosis, monitoring for treatment response, and surveillance for recurrence. This review highlights the recent advancements in the development of biomarkers and their efficacy for the detection of GBM.

https://thejns.org/doi/abs/10.3171/2017.3.JNS162280

KEY WORDS glioblastoma; biomarker; biofluids; diagnosis; oncology

$\mathrm{B}$ IOFLuID-based detection of glial tumors offers multiple approaches for improving quality of life in patients with glioblastoma (GBM). Many screening approaches take advantage of a slow initial phase of tumor growth for early detection, before a tumor has reached a greater degree of malignant potential. For example, in the more common malignancies such as breast and colon cancer, the early discovery of solid tumors with the development of mammography and colonoscopy screenings is a well-established clinical paradigm. In terms of malignant glial tumors (such as GBM), this analogy would extend to the concept of detecting a low-grade tumor prior to its progression to a high-grade glioma (secondary GBM). In patients with GBMs that form de novo (primary GBMs), biofluid screening would likely not be beneficial, and would instead be used in assessing prognosis, therapeutic monitoring, and surveillance of recurrent disease.

To extend survival and improve monitoring of patients with brain tumors, we need a detection strategy that provides 3 independent assessments: the ability to detect tumors early in the disease process, the ability to monitor current therapeutic modalities, and the ability to detect recurrence. Despite the great utility of imaging in evaluating GBM patients, the ongoing limitations of imaging to definitively diagnose and monitor both primary and secondary GBMs points to the need for more cost-efficient and effective biomarkers. ${ }^{34}$ Thus, researchers and clinicians are pursuing serum and CSF biomarkers to detect GBM early enough to provide timely treatment in secondary GBMs, which could potentially enable greater survival benefit, and enable one to follow disease progression and monitor chemotherapy and radiation treatments in primary GBMs.

\section{Rationale for Biomarker Development}

Analyzing markers of malignancy in biofluids was initially established in colorectal cancer with the detection of elevated serum carcinoembryonic antigen levels. ${ }^{16}$ However, given that levels of this normal physiological protein were not always elevated, and that it is associated with various other types of cancer, its use as a diagnostic biomarker is limited. Biomarkers for other cancers soon followed, such as prostate-specific antigen for prostate cancer and cancer antigen 125 for ovarian cancer, but analyses using these markers fail to meet diagnostic test requirements due to relatively low specificity. ${ }^{4,48}$ There are currently more than 20 FDA-approved biomarkers for tumors, the majority of which are only used to monitor response to therapy and progression of disease. ${ }^{15}$ Currently, GBM does not have a reliable biomarker in the serum or CSF, and initial detection relies on clinical diagnosis after the presentation of symptoms caused by the location of the tumor within the brain and its relative size (i.e., aphasia, seizures, paralysis, etc.). Thus, the ability to detect GBM early is vital

ABBREVIATIONS CTC = circulating tumor cell; ctDNA = circulating tumor DNA; EGFR = epidermal growth factor receptor; EM = extracellular macromolecule; EV = extracellular vesicle; GBM = glioblastoma; GFAP = glial fibrillary acidic protein; HGG = high-grade glioma; IDH1 = isocitrate dehydrogenase 1; IL = interleukin; LOH = loss of heterozygosity; MBP = myelin basic protein; MGMT = $0^{6}$-methylguanine methyltransferase; miRNA = microRNA; PTEN = phosphatase and tensin homolog; T-reg = regulatory T cell; VEGF = vascular endothelial growth factor; 2-HG = 2-hydroxyglutarate.

SUBMITTED August 30, 2016. ACCEPTED March 17, 2017.

INCLUDE WHEN CITING Published online October 20, 2017; DOI: 10.3171/2017.3.JNS162280. 
for clinicians to initiate treatment before patients become symptomatic, likely improving progression-free survival and quality of life.

In a future paradigm of GBM therapeutic management, early detection may be beneficial in 2 ways. First, the tumor volume would be smaller if discovered early, resulting in a smaller resection cavity and less impact on adjacent structures compared with the resection of a larger tumor. Second, smaller tumors may have less genetic variation between tumor stem cells and progeny, which can have a significant impact on sensitivity to chemotherapy and radiation. ${ }^{3,36}$ Cells within larger tumors are more genetically diverse, which can facilitate the evasion of immune responses, resistance to therapy, and initiation of recurrence. With regard to the clinical relevance of early detection of smaller tumors, there are studies both supporting and refuting the claim that tumor size correlates with prognosis and improved outcomes. ${ }^{6,7,19,40}$ However, more important than the early detection of small tumors may be the ability to diagnosis genetically favorable tumors such as isocitrate dehydrogenase 1 (IDH1) mutant tumors, which often present with seizures ${ }^{12,46}$ regardless of size, and have a long latent period as lower grade tumors. These tumors may be particularly sensitive to the combination of gross-total resection and chemoradiation.

The presence of certain mutations and cell surface markers in GBM may provide targets for detection in the serum and CSF. Given that the brain is bathed in CSF, biomarkers would theoretically be more prevalent in this biofluid compared with serum. Additionally, the bloodbrain barrier may filter out potentially diagnostic macromolecules. The collection of CSF, however, is a more invasive process than blood collection; thus, identifying a biomarker in the serum would be preferable. Because every GBM is genetically distinct, some responding differently to chemotherapy and radiation regimens, the ability to distinguish between the molecular subtypes of GBM to direct tumor-specific therapy is also ideal for a tumor biomarker. GBMs harbor certain aberrations depending on their subtype, many of which can be detected by molecular profiling as well as by the analysis of extracellular vesicles (EVs) and circulating tumor cells (CTCs; Table 1). Thus, the discovery of a reliable "liquid biopsy" utilizing the serum and/or CSF will not only improve the ability to diagnose GBM, but also impact therapeutic strategies and surveillance of recurrence.

\section{Current Evidence for Biomarkers}

\section{Extracellular Macromolecules (nucleic acids, proteins, and metabolites)}

Free nucleic acid species, including tumor-specific genetic aberrations, can be detected in the blood and CSF of patients with GBM, which may be useful in ruling out disease in normal subjects. Additionally, these nucleic acid signatures may be able to differentiate between the various GBM subtypes, provide prognostic information, and guide tumor-specific therapeutic strategies.

The presence of circulating tumor DNA (ctDNA) in the blood of brain tumor patients was recently reported by Lavon et al. and Majchrzak-Celińska et al. ${ }^{24,27}$ As tu- mor cells begin to die, they release intracellular content, including ctDNA, which can be isolated from the serum and can provide molecular genetic information about the malignancy. The Lavon group examined serum samples from 70 patients with either high-grade gliomas or oligodendrogliomas, and found that loss of heterozygosity (LOH), as well as methylation status of tumor suppressor genes, can be determined by analyzing ctDNA. ${ }^{24}$ Specifically, analysis of serum ctDNA was able to detect methylation of $\mathrm{O}^{6}$-methylguanine methyltransferase $(M G M T)$ and phosphatase and tensin homolog (PTEN), as well as $\mathrm{LOH}$ in chromosomes $1 \mathrm{p}, 19 \mathrm{q}$, and $10 \mathrm{q}$. $\mathrm{LOH}$ and/or methylation was detected in $62(89 \%)$ of the samples. When compared with the tissue gold standard, the sensitivity for these serum biomarkers to predict genetic aberrations in the tumor was $51 \%(\mathrm{LOH})$ and $55 \%$ (methylation), with a specificity of $100 \% .{ }^{24}$ Similarly, the Majchrzak-Celińska group analyzed methylation status in MGMT, RASSF1A, p15INK4B, and pIAARF in serum ctDNA of 33 patients with newly diagnosed CNS tumors, including 17 gliomas, 6 meningiomas, and 10 metastatic malignancies. ${ }^{27}$ Of the 17 samples from glioma patients, 12 (71\%) demonstrated methylation in at least 1 of the 4 tumor suppressor genes: $M G M T$ (18\%), RASSFIA (47\%), pI5INK4B (12\%), and pI4ARF (41\%). When compared with the tissue gold standard, the sensitivity and specificity of these serum biomarkers to detect gene methylation were $50 \%$ and $100 \%$, respectively. ${ }^{27}$ The low concentration of tumor-specific ctDNA in the serum is likely responsible for the relatively poor sensitivity of these nucleic acid biomarkers for detecting intracranial tumors, and suggests that analysis of CSF may improve false-negative rates given the difference in concentrations.

Recently, CSF analysis has demonstrated significantly increased concentrations of tumor-specific ctDNA when compared with serum, as reported by Pan et al. and De Mattos-Arruda et al. ${ }^{8,32}$ The Pan group isolated ctDNA from the CSF of 7 patients with solid brain tumors, 6 of whom had detectable tissue-concordant mutations in at least one of the following genes: NF2, AKT1, BRAF, $N R A S, K R A S$, and EGFR. Although the concentration of total ctDNA was less in CSF compared with serum, the concentration of mutation-specific ctDNA was higher in the CSF. ${ }^{32}$ Similarly, the De Mattos-Arruda group investigated ctDNA from the CSF of 12 patients with solid brain tumors, and found that the mutant allelic frequency was significantly higher in CSF compared with serum. Mutations in EGFR, PTEN, ESR1, IDH1, ERBB2, and FGFR2 were readily detected in the CSF ctDNA with a sensitivity of $58 \%$, compared with $0 \%$ for serum. ${ }^{8}$ Together, the results of these studies indicate that analysis of ctDNA in the CSF may be used for early detection of intracranial brain tumors that harbor specific oncogenic mutations.

Due to the inherent heterogeneity of malignancies, not all tumor cells will express the same genetic mutations. Thus, profiling RNA expression in biofluids may provide a more accurate representation of the malignant process. Dong et al. investigated the microRNA (miRNA) profile in the serum of 3 patients with GBM and compared them to 3 age- and sex-matched healthy controls. ${ }^{9}$ Of the 752 serum miRNAs analyzed, they found that the patients with GBM had 115 that were significantly upregulated and 24 
TABLE 1. Biomarker summary: studies investigating various biomarkers in the blood and CSF of patients with GBM

\begin{tabular}{|c|c|c|c|c|}
\hline Authors \& Year & Biofluid & Biomarker & Sensitivity (\%) & Specificity (\%) \\
\hline \multicolumn{5}{|l|}{ EMs (nucleic acids) } \\
\hline \multirow{2}{*}{ Lavon et al., 2010} & \multirow{2}{*}{ Serum } & MGMT \& PTEN methylation & 55 & 100 \\
\hline & & LOH in $1 p, 19 q, 10 q$ & 51 & 100 \\
\hline Majchrzak-Celińska et al., 2013 & Serum & MGMT, RASSF1A, p15INK4B, \& p14ARF methylation & 50 & 100 \\
\hline Pan et al., 2015 & CSF & NF2, AKT1, BRAF, NRAS, KRAS, \& EGFR mutations & NR & NR \\
\hline De Mattos-Arruda et al., 2015 & CSF & EGFR, PTEN, ESR1, IDH1, ERBB2, \& FGFR2 mutations & 58 & NR \\
\hline Dong et al., 2014 & Serum & $\begin{array}{l}\text { Elevated miR-340, miR-576-5p, \& miR-626; decreased let-7g-5p, } \\
\text { miR-7-5p, \& miR-320 }\end{array}$ & NR & NR \\
\hline Tang et al., 2015 & Serum & Elevated miR-185 & NR & NR \\
\hline Lai et al., 2015 & Serum & Elevated miR-210 & NR & NR \\
\hline Yue et al., 2016 & Serum & Decreased miR-205 & NR & NR \\
\hline Teplyuk et al., 2012 & CSF & Elevated miR-10b, miR-21, \& miR-200 & 91 & 99 \\
\hline Drusco et al., 2015 & CSF & Elevated miR-223, miR-451, \& miR-711 & NR & NR \\
\hline \multicolumn{5}{|l|}{ EMs (proteins) } \\
\hline Jung et al., 2007 & Serum & GFAP $>0.05 \mu \mathrm{g} / \mathrm{ml}$ & 76 & 100 \\
\hline Tichy et al., 2016 & Serum & GFAP > $0.01 \mu \mathrm{g} / \mathrm{ml}$ & 85 & 70 \\
\hline Kiviniemi et al., 2015 & Serum & GFAP $>0.014 \mathrm{ng} / \mathrm{ml}$ & 86 & 85 \\
\hline Hormigo et al., 2006 & Serum & Elevated YKL-40 \& MMP-9 & NR & NR \\
\hline Elstner et al., 2011 & Serum & Elevated BMP2, CXCL10, \& HSP70 & 96 & 89 \\
\hline Quaranta et al., 2007 & Serum & Elevated EGFR & NR & NR \\
\hline Szymaś et al., 1986 & CSF & GFAP $>0.04 \mu \mathrm{g} / \mathrm{ml}$ & NR & NR \\
\hline Nakagawa et al., 1994 & CSF & $\mathrm{MBP}>4.0 \mathrm{ng} / \mathrm{ml}$ & NR & NR \\
\hline Sampath et al., 2004 & CSF & Elevated VEGF & NR & NR \\
\hline Shen et al., 2014 & CSF & Elevated IL-6 & NR & NR \\
\hline \multicolumn{5}{|l|}{ EMs (metabolites) } \\
\hline Mörén et al., 2015 & Serum & Elevated cysteine, lysine, \& 2-oxoisocaproic acid metabolites & NR & NR \\
\hline Locasale et al., 2012 & CSF & Elevated 2-HG, histidine, \& tryptophan metabolites & NR & NR \\
\hline \multicolumn{5}{|l|}{ EVs } \\
\hline Noerholm et al., 2012 & Serum & Decreased RPL11, RPS12, TMSL3, \& B2M & NR & NR \\
\hline Manterola et al., 2014 & Serum & Elevated RNU6-1, miR-320, \& miR-574-3p & 87 & 86 \\
\hline \multirow{2}{*}{ Skog et al., 2008} & \multirow{2}{*}{ Serum } & Detection of EGFRvIll & NR & NR \\
\hline & & Elevated angiogenin, FGF- $\alpha$, IL-6, TIMP-1, TIMP-2, \& VEGF & NR & NR \\
\hline Shao et al., 2012 & & Detection of EGFR, EGFRvIII, PDPN, \& IDH1 & 85 & 80 \\
\hline Akers et al., 2013 & Serum & Elevated miR-21 & 87 & 93 \\
\hline Figueroa et al. (unpublished) & CSF & Detection of EGFRvIII & 60 & 98 \\
\hline \multicolumn{5}{|l|}{ Circulating tumor \& immune cells } \\
\hline Sullivan et al., 2014 & Serum & Tumor cells: CD14-positive, CD16-positive, and CD45-positive & NR & NR \\
\hline MacArthur et al., 2014 & Serum & Tumor cells: hTERT probe & NR & NR \\
\hline Fecci et al., 2006 & Serum & $\begin{array}{l}\text { T regulatory cells: CD4-positive, CD25-positive, } \\
\text { CD45RO-positive, FOXP3-positive }\end{array}$ & NR & NR \\
\hline Heimberger et al., 2008 & Serum & T regulatory cells: FOXP3-positive & NR & NR \\
\hline
\end{tabular}

$\mathrm{NR}=$ not reported.

that were significantly downregulated. Specifically, they identified 3 miRNAs that were the most overexpressed (miR-340, miR-576-5p, and miR-626), and 3 miRNAs that were the most underexpressed (let-7g-5p, miR-7-5p, and miR-320). ${ }^{9}$ Other investigators have also shown that miRNA can be used as a serum biomarker for GBM. Tang et al. showed that levels of miR-185 were significantly increased in the serum of 66 patients with glioma, which returned to normal levels after surgery, chemotherapy, and radiation. ${ }^{43}$ Similarly, Lai et al. demonstrated that miR-210 levels in the serum of 136 patients with GBM correlated with tumor grade and patient outcomes. ${ }^{23}$ Conversely, Yue et al. found that significantly decreased levels of miR-205 in the serum of 64 patients with glioma correlated with Karnofsky Performance Scale scores, tumor grade, and overall survival. ${ }^{49}$ Although results from these studies are promising, 
there are no reported investigations into the sensitivity and specificity of individual, or panels of, serum miRNA to diagnose GBM.

CSF analysis of miRNA in patients with GBM has also yielded some intriguing results. Teplyuk et al. found that miR-10b and miR-21 levels in the CSF were significantly increased in 19 patients with GBM. ${ }^{44}$ Additionally, they showed that levels of miR-200 were only elevated in CNS metastases, which could enable clinicians to differentiate those lesions from primary malignancies. This miRNA trio was able to distinguish GBM and metastases from healthy controls with an accuracy of $91 \%-99 \% .^{44}$ Similarly, Drusco et al. demonstrated that elevated levels of miR223, miR-451, and miR-711 in the CSF can be used to distinguished GBM from other CNS tumors. ${ }^{10}$ Together, these studies suggest that CSF profiling of miRNA may not only be useful in diagnosing GBM, but also in distinguishing it from other malignancies in the CNS.

Much like profiling free miRNA, the search for protein biomarkers in GBM focuses on aberrations in levels of normal physiological secreted proteins, given that no tumor-specific proteins have been identified. This approach relies on detecting proteins secreted into the serum or CSF from malignant cells in hopes of identifying levels at which clinicians can reliably diagnose GBM.

The most widely studied protein in the serum of patients with GBM is glial fibrillary acidic protein (GFAP). This protein is an intermediate filament highly expressed in glial cells, and several reports have shown that elevated serum GFAP levels can be used to diagnose glial tumors such as GBM. ${ }^{20,22,45}$ Jung et al. analyzed the serum of 50 patients with GBM and found that GFAP levels greater than $0.05 \mu \mathrm{g} / \mathrm{ml}$ were $76 \%$ sensitive and $100 \%$ specific for diagnosing malignant gliomas. Additionally, this group showed that GFAP was not detectable in the serum of patients with non-GBM. ${ }^{20}$ Similarly, Tichy et al. analyzed the serum of 33 patients with GBM, and found that GFAP levels greater than $0.01 \mu \mathrm{g} / \mathrm{ml}$ resulted in a sensitivity of $85 \%$ and a specificity of $70 \%$ for diagnosing GBM and differentiating between non-GBM brain tumors. ${ }^{45}$ Lastly, Kiviniemi et al. analyzed the serum of 27 patients with high-grade gliomas (HGGs) and found that GFAP levels greater than $0.014 \mathrm{ng} / \mathrm{ml}$ yielded in a sensitivity of $86 \%$ and a specificity of $85 \%$ for diagnosing HGGs, and correlated with a lack of the IDH1 mutation. ${ }^{22}$ Together, these studies indicate that detection of elevated GFAP levels in the serum may be a useful biomarker for GBM diagnosis.

Several other studies have focused on different proteins related to GBM. Hormigo et al. analyzed the serum of 66 patients with active GBM and 10 patients in GBM remission, and found that elevated levels of YKL-40 and MMP9 in the serum correlated with active tumors and decreased overall survival..$^{18}$ Additionally, Elstner et al. showed that the serum protein profile of 23 patients with HGGs had elevated BMP2, CXCL10, and HSP70, yielding a sensitivity of $96 \%$ and a specificity of $89 \%$ for HGG diagnosis. ${ }^{12}$ Lastly, the analysis of epidermal growth factor receptor (EGFR) levels in the serum appeals to GBM investigators because most of these tumors have increased DNA amplifications and RNA expression of wild-type EGFR. ${ }^{5}$ Quaranta et al. analyzed the serum of 35 patients with GBM, and found elevated EGFR levels compared with normal controls, which was inversely correlated with overall survival. ${ }^{33}$ These results indicate that analysis of these protein levels in the serum may provide a panel by which clinicians can reliably diagnose GBM.

Similar to the nucleic acid biomarkers, protein biomarkers in the CSF may be more concentrated than in the serum. Szymaś et al. analyzed the CSF of 72 patients with intracranial tumors, and found that GFAP levels greater than $0.04 \mu \mathrm{g} / \mathrm{ml}$ enabled differentiation from other CNS malignancies and normal controls..$^{42}$ Similarly, myelin basic protein (MBP), which is unique to the CNS, is an intriguing CSF biomarker in patients with GBM. Nakagawa et al. analyzed the CSF of 7 patients with malignant gliomas and showed that MBP levels greater than $4.0 \mathrm{ng} /$ $\mathrm{ml}$ correlated with active malignancies, which decreased after surgery and chemotherapy. ${ }^{30}$ Growth factors and cytokines have also been identified as potential biomarkers in the CSF of patients with GBM. Sampath et al. demonstrated that $90 \%$ of patients with malignant gliomas had elevated vascular endothelial growth factor (VEGF) levels in the CSF, compared with normal controls. ${ }^{35}$ Shen et al. analyzed the levels of 19 tumor-related proteins in the CSF and demonstrated that patients with GBM had significant increases in interleukin (IL)-6 levels compared with lowgrade gliomas and normal controls. ${ }^{38}$ Together, the results of these studies suggest that CSF proteins may have utility as GBM biomarkers.

Investigators have recently started studying metabolomics in the serum and CSF of patients with GBM. Mörén et al. showed that increased levels of the cysteine metabolite in serum was found in patients with GBM, and that increased levels of the lysine and 2-oxoisocaproic acid metabolites in the serum were found in patients with oligodendrogliomas. ${ }^{29}$ Additionally, elevated levels of myoinositol and hexadecanoic acid metabolites in the serum were found to be of prognostic value in predicting longterm survival. ${ }^{29}$ Similarly, Locasale et al. analyzed 124 cellular metabolites in the CSF of 10 patients with malignant gliomas, and demonstrated that metabolites from the amino acid, central carbon, lipid, and pyrimidine metabolism pathways were significantly different compared with normal controls. ${ }^{25}$ Interestingly, levels of 2-hydroxyglutarate (2-HG) were increased in patients with GBM, indicating the presence of the isocitrate dehydrogenase 1 (IDH1) mutation. ${ }^{25}$ Additionally, elevated levels of histidine and tryptophan metabolites in the CSF can be used to differentiate primary GBM from recurrence. ${ }^{25}$ These studies indicate that while metabolomic biomarkers are relatively new in the field of cancer, they provide valuable real-time information about the cellular energy state within the malignant process.

\section{Extracellular Vesicles}

The use of extracellular vesicles (EVs), derived from the serum and CSF, as a biomarker of GBM is not as well studied compared with extracellular macromolecules (EMs). However, free nucleic acids and proteins are prone to more degradation than EVs and their contents, given the relatively harsh extracellular environment in the serum, and even CSF. EV secretion is a normal physiological process that has been described for nearly every type of cell, 
and itself is a broad term that encompasses microvesicles and exosomes..$^{21}$ Microvesicles (200-500 $\mathrm{nm}$ in diameter) are released via cell membrane blebbing and can contain cytoplasmic elements such as mRNA and miRNA. Exosome (40-100 $\mathrm{nm}$ in diameter) packaging and secretion, however, is more regulated and involves the intracellular endosomal system. ${ }^{21}$ Both microvesicles and exosomes can contain tumor-specific elements of the cell membrane, as well as nucleic acid species, which have utility as diagnostic biomarkers when isolated from the serum and CSF of patients with GBM.

EVs are known to contain an appreciable amount of nucleic acid species that can be profiled as biomarkers for GBM. Noerholm et al. analyzed serum-derived EVs from 10 patients with GBM and found significantly lower levels of 4 ribosomal function genes compared with normal controls: RPL11, RPS12, TMSL3, and B2M. ${ }^{31}$ However, no genes were identified in serum-derived EVs that were upregulated in patients with GBM. ${ }^{31}$ Similarly, Skog et al. showed that EGFRvIII mRNA is detectable in serumderived microvesicles, which may direct receptor-specific adjuvant therapy. ${ }^{39}$ Lastly, Manterola et al. found that increased levels of RNU6-1, miR-320, and miR-574-3p correlated with GBM diagnosis, yielding a sensitivity and specificity of $87 \%$ and $86 \%$, respectively. ${ }^{28}$ Together, these studies suggest that RNA profiling of serum-derived EVs can provide detailed transcriptomics of malignant cells to be used in GBM diagnosis.

Serum-derived EVs are also known to contain proteins that have biomarker potential in GBM. Skog et al. found that serum-derived EVs in patients with GBM had increased levels of angiogenin, FGF- $\alpha$, IL-6, TIMP-1, TIMP-2, and VEGF. ${ }^{39}$ This idea of developing a protein expression panel to diagnose GBM was then improved upon by Shao et al. ${ }^{37}$ Using $\mu$ NMR technology, this group analyzed a panel of 4 GBM-related proteins (EGFR, EGFRvIII, PDPN, and IDH1) in serum-derived EVs, yielding a diagnostic sensitivity and specificity of $85 \%$ and $80 \%$, respectively. ${ }^{37}$ Additionally, this protein quad-panel had prognostic value in predicting how patients responded to temozolomide chemotherapy. ${ }^{37}$ Thus, the relative abundance of tumor-related proteins within serum-derived EVs confers considerable diagnostic potential as biomarkers for GBM.

As with free-circulating EMs, CSF-derived EVs are more likely to be GBM-specific compared with those in the serum. Akers et al. analyzed EVs from the CSF of 13 patients with GBM and found an average 10-fold increase in miR-21 levels compared with nononcologic controls. ${ }^{2}$ These results were then validated in a cohort of 29 patients with GBM, in which CSF-derived EVs yielded a diagnostic sensitivity and specificity of $87 \%$ and $93 \%$, respectively. ${ }^{2}$ Subsequently, this group also showed that while CSF-derived microvesicles contain more total RNA, CSFderived exosomes were enriched with miRNA. ${ }^{1}$ Of the 754 miRNAs analyzed in this study, 46 were present only in exosomes, while just 11 miRNAs were present in both microvesicles and exosomes. ${ }^{1}$ The intriguing results of these studies suggest that miRNA profiling of CSF-derived EVs may have utility in GBM diagnosis.

Recently, investigators have also studied CSF-derived
EVs for the presence of GBM-specific genetic mutations. Figueroa et al. ${ }^{14}$ showed that EGFRvIII is detectable in CSF-derived EVs in a subgroup of 81 patients with GBM. Of the 25 patients $(30.8 \%)$ who were EGFRvIII tissuepositive, 15 had detectable levels of EGFRvIII in CSF-derived EVs, yielding a sensitivity of $60 \%$. Of the 56 patients (69.2\%) who were EGFRvIII tissue-negative, only 1 patient had detectable EGFRvIII in CSF-derived EVs, yielding a specificity of $98 \%$. Additionally, EGFRvIII-positive CSF-derived EVs had increased mRNA expression of wild-type $E G F R$, which is a known aberration present in the classical subtype of GBM. ${ }^{47}$ Given these results, this novel diagnostic modality has potential for diagnosing a subpopulation of GBM via EGFRvIII status, as well as differentiating a GBM subtype.

\section{Circulating Tumor and Immune Cells}

The least researched approach to diagnosing GBM via biofluids is detecting CTCs. This strategy involves isolating primarily rogue tumor cells that separate from the primary malignant mass and enter the blood or CSF, but can also include subverted tumor stromal cells, such as immune and endothelial cells. Currently, CTCs have only been studied in the serum of patients with GBM, and no investigations identifying CTCs in the CSF have been reported.

Searching for CTCs in the serum of patients with GBM is difficult given that reliable tumor-specific cell surface markers have yet to be established. Thus, investigators have targeted panels of membrane proteins that may identify GBM-specific CTCs. Using a trio of antibodies (anti-CD14, anti-CD16, and anti-CD45) Sullivan et al. detected CTCs in the serum of 13 of 33 patients with GBM. ${ }^{41}$ CTC status was subsequently confirmed by FISH analysis using genetic aberrations known to be present in specific GBM subtypes: proneural (ASCL1, DLL3, OLIG2, and SOX2), neural (SLC12A5 and SYT1), classic (AKT2, EGFR, and GFAP), and mesenchymal (RELB, SERPINE1, and $T G F B 1){ }^{41}$ Similarly, MacArthur et al. isolated CTCs from the serum of 8 of 11 patients with GBM using an adenoviral probe to $h T E R T$, which is elevated in this malignancy. ${ }^{26} \mathrm{CTC}$ status was subsequently confirmed by FISH analysis for the overexpression of wild-type EGFR, GFAP, and nestin, and the absence of EpCAM. ${ }^{26}$ The promising results of these studies indicate that detecting CTCs in the serum is possible in patients with GBM, and may prove to be a viable diagnostic strategy in the future.

The detection of tumor-associated immune cells in the blood has also been described in patients with GBM. Immune system evasion and manipulation are hallmarks in cancer, and are well described in malignant glioma. Several groups have focused their efforts on identifying the increasing regulatory T-cell (T-reg) population in the peripheral blood of patients with GBM. T-regs are known as immune system suppressors and may act to decrease the immune response to a growing malignancy. Fecci et al. showed that while absolute counts of CD4-positive T cells and T-regs decreases over time in patients with GBM, the fraction of T-regs in the remaining CD4-positive cells increases in the blood. ${ }^{13}$ Similarly, Heimberger et al. found that T-regs were not present in regular brain or low-grade gliomas, but only in malignant gliomas, suggesting that 
their presence is detectable in the serum. ${ }^{17}$ Together, these studies indicate that evaluating a patient's serological immune response to GBM could be a method of monitoring and/or diagnosing HGGs.

\section{Summary}

Analyzing biofluids to diagnose GBM earlier in the malignant process is an ever-evolving area of interest in neurooncology. Investigators have discovered numerous free nucleic acid and protein signatures in the serum and CSF that are paving the way for diagnostic biomarkers. The field of EV isolation and content characterization is expanding and improving, providing another potentially diagnostic approach. Finally, additional research is required to more effectively detect CTCs, which have proven to be an intriguing and promising modality for GBM diagnosis. These novel approaches may not only provide tumor-specific information that can direct individualized therapeutic strategies, but may also be used to screen for secondary GBMs, monitor therapeutic efficacy in primary GBMs, and survey for recurrent disease. However, to reach the stage of a practical and effective assay for detection of GBM, greater sensitivity and specificity of newer "liquid biopsy" techniques is required. Additionally, because obtaining CSF involves a semi-invasive procedure, the "holy grail" would be to develop a blood-based assay that reduces patient risk. If a low-cost and robust serum biomarker can be identified, then early diagnosis of secondary GBM is viable, and active monitoring of both primary and secondary GBM would be facilitated. For now, current assays are limited in scope, although with the demand and funding for such diagnostic and screening tools increasing, patients may be able to benefit from these advances in the near future.

\section{References}

1. Akers JC, Ramakrishnan V, Kim R, Phillips S, Kaimal V, Mao Y, et al: miRNA contents of cerebrospinal fluid extracellular vesicles in glioblastoma patients. J Neurooncol 123:205-216, 2015

2. Akers JC, Ramakrishnan V, Kim R, Skog J, Nakano I, Pingle $\mathrm{S}$, et al: MiR-21 in the extracellular vesicles (EVs) of cerebrospinal fluid (CSF): a platform for glioblastoma biomarker development. PLoS One 8:e78115, 2013

3. Aubry M, de Tayrac M, Etcheverry A, Clavreul A, Saikali S, Menei P, et al: From the core to beyond the margin: a genomic picture of glioblastoma intratumor heterogeneity. Oncotarget 6:12094-12109, 2015

4. Bast RC Jr, Klug TL, St John E, Jenison E, Niloff JM, Lazarus $\mathrm{H}$, et al: A radioimmunoassay using a monoclonal antibody to monitor the course of epithelial ovarian cancer. $\mathbf{N}$ Engl J Med 309:883-887, 1983

5. Benito R, Gil-Benso R, Quilis V, Perez M, Gregori-Romero M, Roldan P, et al: Primary glioblastomas with and without EGFR amplification: relationship to genetic alterations and clinicopathological features. Neuropathology 30:392-400, 2010

6. Chaichana KL, Chaichana KK, Olivi A, Weingart JD, Bennett R, Brem H, et al: Surgical outcomes for older patients with glioblastoma multiforme: preoperative factors associated with decreased survival. Clinical article. J Neurosurg 114:587-594, 2011

7. Chaichana KL, Martinez-Gutierrez JC, De la Garza-Ramos
R, Weingart JD, Olivi A, Gallia GL, et al: Factors associated with survival for patients with glioblastoma with poor preoperative functional status. J Clin Neurosci 20:818-823, 2013

8. De Mattos-Arruda L, Mayor R, Ng CK, Weigelt B, MartínezRicarte F, Torrejon D, et al: Cerebrospinal fluid-derived circulating tumour DNA better represents the genomic alterations of brain tumours than plasma. Nat Commun 6:8839, 2015

9. Dong L, Li Y, Han C, Wang X, She L, Zhang H: miRNA microarray reveals specific expression in the peripheral blood of glioblastoma patients. Int J Oncol 45:746-756, 2014

10. Drusco A, Bottoni A, Laganà A, Acunzo M, Fassan M, Cascione L, et al: A differentially expressed set of microRNAs in cerebro-spinal fluid (CSF) can diagnose CNS malignancies. Oncotarget 6:20829-20839, 2015

11. Elstner A, Stockhammer F, Nguyen-Dobinsky TN, Nguyen QL, Pilgermann I, Gill A, et al: Identification of diagnostic serum protein profiles of glioblastoma patients. J Neurooncol 102:71-80, 2011

12. Faguer R, Tanguy JY, Rousseau A, Clavreul A, Menei P: Early presentation of primary glioblastoma. Neurochirurgie 60:188-193, 2014

13. Fecci PE, Mitchell DA, Whitesides JF, Xie W, Friedman $\mathrm{AH}$, Archer GE, et al: Increased regulatory T-cell fraction amidst a diminished CD4 compartment explains cellular immune defects in patients with malignant glioma. Cancer Res 66:3294-3302, 2006

14. Figueroa JM, Skog J, Akers J, Li H, Komotar R, Jensen R, et al: Detection of wild-type EGFR amplification and EGFRvIII mutation in CSF-derived extracellular vesicles of glioblastoma patients. Neuro Oncol [epub ahead of print], 2017

15. Füzéry AK, Levin J, Chan MM, Chan DW: Translation of proteomic biomarkers into FDA approved cancer diagnostics: issues and challenges. Clin Proteomics 10:13, 2013

16. Gold P, Freedman SO: Specific carcinoembryonic antigens of the human digestive system. J Exp Med 122:467-481, 1965

17. Heimberger AB, Abou-Ghazal M, Reina-Ortiz C, Yang DS, Sun W, Qiao W, et al: Incidence and prognostic impact of $\mathrm{FoxP}^{+}$regulatory $\mathrm{T}$ cells in human gliomas. Clin Cancer Res 14:5166-5172, 2008

18. Hormigo A, Gu B, Karimi S, Riedel E, Panageas KS, Edgar MA, et al: YKL-40 and matrix metalloproteinase-9 as potential serum biomarkers for patients with high-grade gliomas. Clin Cancer Res 12:5698-5704, 2006

19. Jeswani S, Nuño M, Folkerts V, Mukherjee D, Black KL, Patil CG: Comparison of survival between cerebellar and supratentorial glioblastoma patients: surveillance, epidemiology, and end results (SEER) analysis. Neurosurgery 73:240-246, 2013

20. Jung CS, Foerch C, Schänzer A, Heck A, Plate KH, Seifert $\mathrm{V}$, et al: Serum GFAP is a diagnostic marker for glioblastoma multiforme. Brain 130:3336-3341, 2007

21. Kalra H, Simpson RJ, Ji H, Aikawa E, Altevogt P, Askenase P, et al: Vesiclepedia: a compendium for extracellular vesicles with continuous community annotation. PLoS Biol 10:e1001450, 2012

22. Kiviniemi A, Gardberg M, Frantzén J, Parkkola R, Vuorinen V, Pesola M, et al: Serum levels of GFAP and EGFR in primary and recurrent high-grade gliomas: correlation to tumor volume, molecular markers, and progression-free survival. J Neurooncol 124:237-245, 2015

23. Lai NS, Wu DG, Fang XG, Lin YC, Chen SS, Li ZB, et al: Serum microRNA-210 as a potential noninvasive biomarker for the diagnosis and prognosis of glioma. Br J Cancer 112: $1241-1246,2015$

24. Lavon I, Refael M, Zelikovitch B, Shalom E, Siegal T: Serum DNA can define tumor-specific genetic and epigenetic markers in gliomas of various grades. Neuro Oncol 12:173-180, 2010 
25. Locasale JW, Melman T, Song S, Yang X, Swanson KD, Cantley LC, et al: Metabolomics of human cerebrospinal fluid identifies signatures of malignant glioma. Mol Cell Proteomics 11:M111.014688, 2012

26. MacArthur KM, Kao GD, Chandrasekaran S, Alonso-Basanta M, Chapman C, Lustig RA, et al: Detection of brain tumor cells in the peripheral blood by a telomerase promoter-based assay. Cancer Res 74:2152-2159, 2014

27. Majchrzak-Celińska A, Paluszczak J, Kleszcz R, Magiera M, Barciszewska AM, Nowak S, et al: Detection of MGMT, RASSF1A, p15INK4B, and p14ARF promoter methylation in circulating tumor-derived DNA of central nervous system cancer patients. J Appl Genet 54:335-344, 2013

28. Manterola L, Guruceaga E, Gállego Pérez-Larraya J, González-Huarriz M, Jauregui P, Tejada S, et al: A small noncoding RNA signature found in exosomes of GBM patient serum as a diagnostic tool. Neuro Oncol 16:520-527, 2014

29. Mörén L, Bergenheim AT, Ghasimi S, Brännström T, Johansson M, Antti H: Metabolomic screening of tumor tissue and serum in glioma patients reveals diagnostic and prognostic information. Metabolites 5:502-520, 2015

30. Nakagawa H, Yamada M, Kanayama T, Tsuruzono K, Miyawaki Y, Tokiyoshi K, et al: Myelin basic protein in the cerebrospinal fluid of patients with brain tumors. Neurosurgery 34:825-833, 1994

31. Noerholm M, Balaj L, Limperg T, Salehi A, Zhu LD, Hochberg FH, et al: RNA expression patterns in serum microvesicles from patients with glioblastoma multiforme and controls. BMC Cancer 12:22, 2012

32. Pan W, Gu W, Nagpal S, Gephart MH, Quake SR: Brain tumor mutations detected in cerebral spinal fluid. Clin Chem 61:514-522, 2015

33. Quaranta M, Divella R, Daniele A, Di Tardo S, Venneri MT, Lolli I, et al: Epidermal growth factor receptor serum levels and prognostic value in malignant gliomas. Tumori 93:275280, 2007

34. Raizer JJ, Fitzner KA, Jacobs DI, Bennett CL, Liebling DB, Luu TH, et al: Economics of malignant gliomas: a critical review. J Oncol Pract 11:e59-e65, 2015

35. Sampath P, Weaver CE, Sungarian A, Cortez S, Alderson L, Stopa EG: Cerebrospinal fluid (vascular endothelial growth factor) and serologic (recoverin) tumor markers for malignant glioma. Cancer Contr 11:174-180, 2004

36. Shackleton M, Quintana E, Fearon ER, Morrison SJ: Heterogeneity in cancer: cancer stem cells versus clonal evolution. Cell 138:822-829, 2009

37. Shao H, Chung J, Balaj L, Charest A, Bigner DD, Carter BS, et al: Protein typing of circulating microvesicles allows realtime monitoring of glioblastoma therapy. Nat Med 18:18351840,2012

38. Shen F, Zhang Y, Yao Y, Hua W, Zhang HS, Wu JS, et al: Proteomic analysis of cerebrospinal fluid: toward the identification of biomarkers for gliomas. Neurosurg Rev 37:367380,2014

39. Skog J, Würdinger T, van Rijn S, Meijer DH, Gainche L, Sena-Esteves M, et al: Glioblastoma microvesicles transport RNA and proteins that promote tumour growth and provide diagnostic biomarkers. Nat Cell Biol 10:1470-1476, 2008
40. Song KS, Phi JH, Cho BK, Wang KC, Lee JY, Kim DG, et al: Long-term outcomes in children with glioblastoma. J Neurosurg Pediatr 6:145-149, 2010

41. Sullivan JP, Nahed BV, Madden MW, Oliveira SM, Springer $\mathrm{S}$, Bhere D, et al: Brain tumor cells in circulation are enriched for mesenchymal gene expression. Cancer Discov 4:1299-1309, 2014

42. Szymaś J, Morkowski S, Tokarz F: Determination of the glial fibrillary acidic protein in human cerebrospinal fluid and in cyst fluid of brain tumors. Acta Neurochir (Wien) 83:144150,1986

43. Tang H, Liu Q, Liu X, Ye F, Xie X, Xie X, et al: Plasma miR185 as a predictive biomarker for prognosis of malignant glioma. J Cancer Res Ther 11:630-634, 2015

44. Teplyuk NM, Mollenhauer B, Gabriely G, Giese A, Kim E, Smolsky M, et al: MicroRNAs in cerebrospinal fluid identify glioblastoma and metastatic brain cancers and reflect disease activity. Neuro Oncol 14:689-700, 2012

45. Tichy J, Spechtmeyer S, Mittelbronn M, Hattingen E, Rieger J, Senft C, et al: Prospective evaluation of serum glial fibrillary acidic protein (GFAP) as a diagnostic marker for glioblastoma. J Neurooncol 126:361-369, 2016

46. Toledo M, Sarria-Estrada S, Quintana M, Maldonado X, Martinez-Ricarte F, Rodon J, et al: Epileptic features and survival in glioblastomas presenting with seizures. Epilepsy Res 130:1-6, 2017

47. Verhaak RG, Hoadley KA, Purdom E, Wang V, Qi Y, Wilkerson $\mathrm{MD}$, et al: Integrated genomic analysis identifies clinically relevant subtypes of glioblastoma characterized by abnormalities in PDGFRA, IDH1, EGFR, and NF1. Cancer Cell 17:98-110, 2010

48. Vihko P, Sajanti E, Jänne O, Peltonen L, Vihko R: Serum prostate-specific acid phosphatase: development and validation of a specific radioimmunoassay. Clin Chem 24:19151919, 1978

49. Yue X, Lan F, Hu M, Pan Q, Wang Q, Wang J: Downregulation of serum microRNA-205 as a potential diagnostic and prognostic biomarker for human glioma. J Neurosurg 124:122-128, 2016

\section{Disclosures}

The authors report no conflict of interest concerning the materials or methods used in this study or the findings specified in this paper.

\section{Author Contributions}

Drafting the article: both authors. Critically revising the article: both authors. Reviewed submitted version of manuscript: both authors. Approved the final version of the manuscript on behalf of both authors: Carter.

\section{Correspondence}

Bob S. Carter, Massachusetts General Hospital, Department of Neurosurgery, GRB 502, 55 Fruit St., Boston, MA 02114. email: bcarter@mgh.harvard.edu. 